\section{$\mathbf{M} / \mathbf{S}$}

médecine/sciences $1988 ; 5: 272-273$

\title{
LES NEUROPEPTIDES DE L'AXE TUBE DIGESTIF-SYSTĖME NERVEUX CENTRAL
}

\section{Dominique Bataille}

Directeur de recherche à l'Inserm

\section{RÉFÉRENCES}

1. Dubois MP. Immunoreactive somatostatin is present in discrete cells of the endocrine pancreas. Proc Natl Acad Sci USA $1975 ; 72: 1340-3$.

2. Vanderhaegen JJ, Signeau JC, Gepts W. New peptide in the vertebrate CNS reacting with antigastrin antibodies. Nature 1975 ; 257 : 603-5.

3. Said SI. Vasoactive intestinal peptide. Advances in peptide hormone research series. New York: Raven Press, 1982 ; 512.

4. Blache P, Kervran A, Bataille D. Oxyntomodulin and glicentin: brain-gut peptides. Regul Pept $1987 ; 18$ : 349.

\section{ADRESSE}

D. Bataille : centre Cnrs-Inserm de pharmacologie-endocrinologie, 34094 Montpellier Cedex,

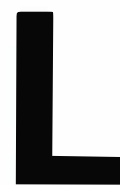

es peptides gastro-intestinaux, messagers chimiques du tube digestif, jouent un rôle fondamental dans le contrôle des fonctions de nutrition (digestion et absorption des nutriments, sécrétions d'enzymes, d'eau et d'électrolytes, motricité digestive, etc.). Sur un plan général, les deux concepts principaux de messager chimique, hormones et neuro-transmetteurs, étaient parfaitement individualisés il y a encore une quinzaine d'années : les premières, stéroïdes et peptides, évoluent dans un domaine, le plasma sanguin périphérique, séparé du système nerveux central (SNC) par la barrière hématoencéphalique ; les seconds, distincts des premiers sur le plan chimique, naissent, agissent et meurent dans un domaine réservé, présentant peu de connexions avec le premier. Ces deux circuits, parallèles et complémentaires, forment la base moléculaire de la régulation intercellulaire. Une relation entre peptides et SNC existe néanmoins au travers des facteurs peptidiques qui contrôlent la libération des hormones hypophysaires. Cependant, chaque molécule peptidique (hormone, neuro-hormone ou facteur de libération d'autres hormones) paraissait initialement très spécialisée, son lieu de synthèse et de sécrétion dans l'organisme correspondant parfaitement à son rôle, apparemment unique.

Ce schéma, qui sépare de manière nette le domaine «périphérique » du domaine «central », a subi depuis 1975 une véritable révolution à la suite de plusieurs observations : Dubois [1], en France, remarque que de la somatostatine, peptide hypothalamique isolé par l'équipe de Guillemin comme inhibiteur de la libération d'hormone de croissance, est présente dans des cellules spécialisées des îlots de Langerhans du pancréas. A la même époque, une équipe belge [2], suivie par d'autres, observent la présence, au sein du système nerveux central, d'une immunoréactivité de type

\section{RÉFÉRENCES}

5. Gluschankof $P$, Cohen $P$. Proteolytic enzymes in the posttranslational processing of polypeptide hormone precursors. Neurochem Res 1987 ; 12: 9518.

6. Bataille D, Blache P, Mercier $F$, et al. Glucagon and related peptides : molecular structure and biological specificity. Ann NY Acad Sci 1987 (sous presse).

7. Edvinsson L, Hakanson R, Wahlestedt C, Uddman R. Effects of neuropeptide $\mathrm{Y}$ on the cardiovascular system. Trends Pharmacol Sci 1987; 8: 231-5.

8. Philips AG, Lane RF, Blaha CD. Inhibition of dopamine release by cholecystokinin : relevance to schizophrenia. Trends Pharmacol Sci 1986 ; 7 : 126-7.

9. Grider JR, Makhlouf GM Colonic peristaltic reflex : Identification of vasoactive intestinal peptide as mediator of descending relaxation. $A m J$ Physiol 1986 ; 251 : G40-5.

10. Dubrasquet JM, AudoussetPuech MP, Martinez J, Bataille D. Somatostatin enhances the inhibitory effect of oxyntomodulin and its C-terminal octapeptide on acid secretion. Peptides 1986; 7 (suppl. 1) : 257-9. 
gastrine ou cholécystokinine (CCK), hormones produites par des cellules endocrines de la muqueuse digestive. Cette immunoréactivité est liée à l'octapeptide C-terminal de la CCK. Un autre peptide qui a marqué l'histoire des relations entre hormones digestives et SNC est le vasoactive intestinal peptide (VIP). Isolé par Said et Mutt de l'intestin de porc comme molécule à propriétés vasoactives [3] et dont des récepteurs, couplés à l'adénylate cyclase, ont été rapidement découverts (voirl'article de G. Rosselin dans ce numéro, p. 287), il a été localisé au sein de corps cellulaires, de fibres et de terminaisons nerveuses des couches sous-muqueuse et musculeuses de l'intestin, et dans le SNC [3]. Outre la CCK et le VIP, on peut citer d'autres peptides co-localisés dans le tube digestif et dans diverses structures du SNC, tels les neurokinines (substance $\mathrm{P}$ et peptides apparentés), la neurotensine, les endorphines, la somatostatine, le calcitonin-gene related peptide (CGRP), les peptides apparentés au polypeptide pancréatique (PYY et NPY), la galanine ou les peptides dérivant du pré-proglucagon [4]. La liste est fort longue et s'allongera encore, la plupart des peptides digestifs étant retrouvés dans diverses structures du SNC.

Les données actuelles concernant le rôle et le mode d'action de ces peptides, tant au niveau périphérique que central, aboutissent à un certain nombre de concepts fondamentaux. (a) Les trois types de voies par lesquelles les messagers chimiques agissent (endocrine, paracrine et neurocrine) sont mis en jeu. (b) Plusieurs formes moléculaires dérivent d'un précurseur commun dont la maturation post-traductionnelle [5], souvent distincte d'un tissu à l'autre [6], aboutit à des spécificités d'organes. Par exemple, dans le SNC, la forme moléculaire principale de CCK est l'octapeptide C-terminal, alors que des formes plus longues dominent dans $\mathrm{m} / \mathrm{s} n^{\circ} 5$ vol. 4, mai 88 les cellules endocrines de l'intestin et dans le plasma (CCK-33, 39 ou 58). De même, la somatostatine- 14 est essentiellement liée à des structures nerveuses et/ou paracrines, la forme longue (somatostatine 28), produite dans l'intestin, ayant un profil de type endocrine. A l'inverse, le même type de maturation post-traductionnelle peut exister dans des cellules endocrines intestinales et dans l'hypothalamus, comme pour les peptides de la famille du glucagon [4]. (c) En relation avec ces formes moléculaires, l'existence de sous-classes de récepteurs permet aux diverses formes moléculaires d'exprimer leur spécificité. Il existe, par exemple, des récepteurs « centraux » de la CCK, présentant une pharmacologie différente de celle des récepteurs « périphériques ». Les différents types de récepteurs des endorphines $(\mu, \delta$, etc. $)$ sont, quant à eux, bien caractérisés. Dans la famille du glucagon, des systèmes de reconnaissance spécifiques de chaque fragment peptidique, couplés à des systèmes de seconds messagers différents (AMP cyclique, phosphoinositides, pompe à calcium, etc.) ont été mis en évidence [6]. (d) Les peptides présents dans des structures nerveuses sont souvent co-synthétisés et co-libérés avec des neurotransmetteurs classiques. Les études menées par diverses équipes, et en particulier celles de l'école suédoise [7] indiquent qu'il existe un véritable partenariat entre le peptide et le neurotransmetteur correspondant. Par exemple le NPY et la noradrénaline (NA) se trouvent dans les mêmes fibres du système sympathique. Le peptide agirait à la fois comme un neuromodulateur (via des récepteurs pré-synaptiques inhibiteurs de la libération de la NA et via des récepteurs post-synaptiques inhibiteurs de l'effet du neurotransmetteur) et comme un véritable neurotransmetteur via des récepteurs postsynaptiques activateurs. La spécificité de la réponse de la fibre (libéra- tion du peptide ou du neurotransmetteur) dépend de la fréquence et du rythme de la stimulation électrique appliquée. Il y a là les bases d'un contrôle physiologique fin grâce à un équilibre entre la libération, à partir d'une même fibre nerveuse, de deux substances à effets antagonistes et/ou complémentaires, suivant l'état de stimulation d'un neurone en amont. Ce type de relation peut sans doute être étendu à d'autres couples peptidesneurotransmetteurs, comme CCK et dopamine, dont les déséquilibres ont été mis en cause dans la schizophrénie [8] ou VIP et acétylcholine dont le jeu de balance est impliqué dans le contrôle du péristaltisme intestinal [9]. D'autres types de partenariat existent probablement (par exemple : hormone-hormone ou hormone-peptide à sécrétion paracrine) via des effets de potentialisation ou d'antagonisme entre molécules complémentaires [10]. Ce jeu fait partie intégrante des mécanismes de régulation biologique.

L'évolution rapide des connaissances fondamentales dans ce domaine foisonnant des peptides régulateurs, grâce aux progrès effectués dans les méthodes de purification (isolement de nouveaux peptides), de biologie cellulaire (mode d'action) et de biologie moléculaire (caractérisation des précurseurs, régulation génomique), doit déboucher sur deux types d'application : sur le plan médical, les interfaces recherche fondamentalerecherche clinique fonctionnant dans les deux sens doivent permettre d'appliquer les connaissances sur les peptides régulateurs aux maladies digestives; dans le domaine de la pharmacologie, les interfaces chimie-biologie et recherche-industrie doivent se nourrir des progrès effectués dans les domaines de la chimie de synthèse et de la conception moléculaire assistée par ordinateur, permettant de faire dériver de peptides régulateurs certains des médicaments de demain 